Revista de Dialectología y Tradiciones Populares, vol. LXXII, n. ${ }^{\circ}$ 1, pp. 59-66, enero-junio 2017, ISSN: 0034-7981, eISSN: 1988-8457, doi: $10.3989 /$ rdtp.2017.01.001.07

\title{
Dejarlo todo y empezar desde cero: el caso de muchos
}

\section{To Leave Everything Behind and Start from Scratch: The Case of Many}

\author{
Núria Matencio Boixeda ${ }^{1}$ \\ Arquitecta Técnica \\ Marta M. Vilela ${ }^{2}$ \\ Fotógrafa Profesional
}

\section{RESUMEN}

¿Cuáles con los motivos que empujan a los jóvenes a buscar oportunidades fuera de su país? ¿Es el contexto social-económico de crisis la razón principal? ¿Qué espera la sociedad de estos jóvenes? A estas alturas muchos son los jóvenes que nos pueden hablar de su experiencia y con cada uno las respuestas serán distintas. Este artículo plantea a modo de experiencia etnográfica lo que significa empezar desde cero en un entorno distinto. Habla de tomar las riendas solo en otro país, de miedos, dudas y perseverancia sin olvidarnos de la convivencia entre la sed por lo nuevo y la añoranza por lo de siempre. Habla de las emociones encontradas durante el proceso e incluso cuando tienes la sensación de haberlo conseguido, ya que, seguramente, lo más difícil acaba siendo decidir volver y, finalmente, volver.

Palabras clave: Emigración juvenil; Retorno; Autobiografía.

\section{SUMMARY}

Why do young people leave their countries to find new opportunities? Do they leave for socio-economic reasons? Is this what society expects of them? Nowadays, many young people can tell us about their experiences and each may have a different story. This article explores the ethnographic experience of starting from scratch in a new and different place. It talks about taking

\footnotetext{
${ }^{1}$ Correo electrónico: Nuria.matencio@gmail.com. ORCID ID: http://orcid.org/0000-0002-3653-4309.

${ }^{2}$ Correo electrónico: Martamvilela@gmail.com. ORCID ID: http://orcid.org/000-0002-3598-2433. Autora de las fotografías del texto.
} 
control, alone, in another country with fears, doubts and perseverance, without forgetting the coexistence of thirst for the new and yearning for the usual. It tells of conflicting emotions throughout the journey, even when you feel you have achieved what you were looking for, because the most difficult of these is probably deciding to return and ultimately returning.

Key words: Youth Migration; Return; Autobiography.

"He mejorado el nivel de inglés y trabajado de lo que he estudiado, en Londres". Estas afirmaciones son suficientes para que la gente no ponga cara de decepción y, por lo general, entienda que emigrar sirvió de algo.

Mi caso es el de una persona joven formada en la universidad apostando por una profesión. Una joven que vuelve a estudiar para ampliar el abanico de opciones, que compagina continuamente estudios, trabajo y voluntariado. Una que fue niña imaginándose "de mayor", que de repente se encuentra en medio de un camino que no va a ninguna parte, aparentemente; un camino en el que por mucho que hagas, insistas y sigas, parece que apuntas a nada porque nada es lo que hay.

Y entonces te vas, para seguir intentándolo.

En relación con lo anterior, tenemos a generaciones anteriores preocupadas porque "tenemos que irnos", olvidando por un momento (ellos no olvidan, pero nosotros sí), por todo lo que ellos han pasado, para conseguir sobrevivir o conseguir lo que perseguían. Hay que recordar las dificultades sociales de sobrevivir a una guerra y/o la posguerra, el exilio o campos de concentración, el dar de comer a tus hijos y todo lo que puedas para que no les falte de nada en una sociedad que cada vez quiere más.

En otras palabras, muchas personas a lo largo de la historia han luchado y nosotros no vamos a ser menos. Cambian las

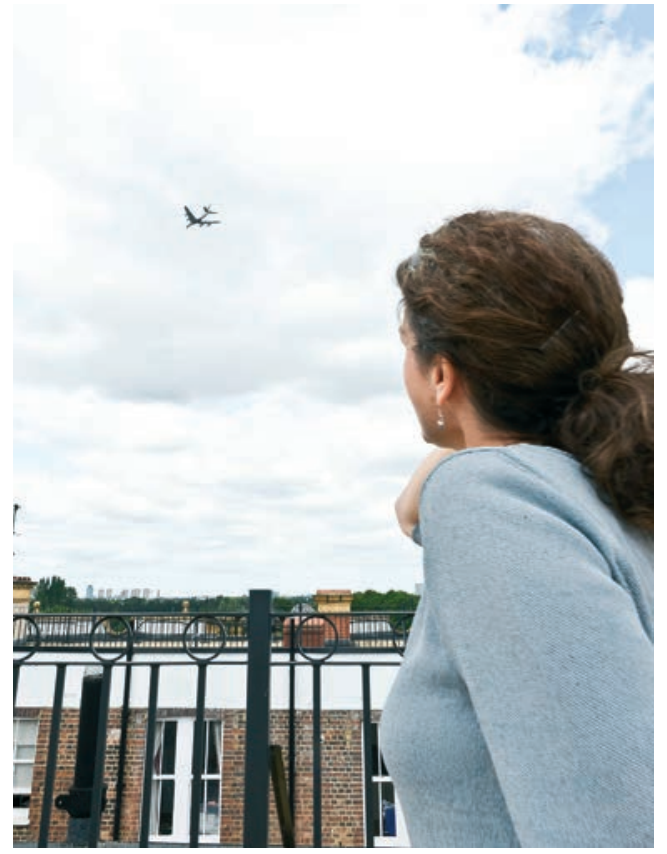
circunstancias sociales y personales, pero todos en algún momento, si no siempre, tenemos que luchar por la vida y nuestros sueños.

Y toda esta introducción, ¿para qué? Nada más lejos que el hecho de dejar de buscar culpables. Al final, el único culpable es aquel que busca, incansable, algo más o diferente, conlleve lo que conlleve.

Porque sí, es duro. La experiencia de dejarlo todo (lleves contigo lo que te lleves, en el fondo lo dejas todo), es un salto al vacío lleno de incertidumbre.

Porque sí, es tan duro como excitante el momento en que aterrizas con el avión sólo contigo mismo y las maletas. Es un momento en que sientes mucho, pero sin saber exactamente qué. Así que sencillamente te dedicas a seguir el plan que te has marcado, que suele ser el de llegar al sitio donde decidiste alojarte o 


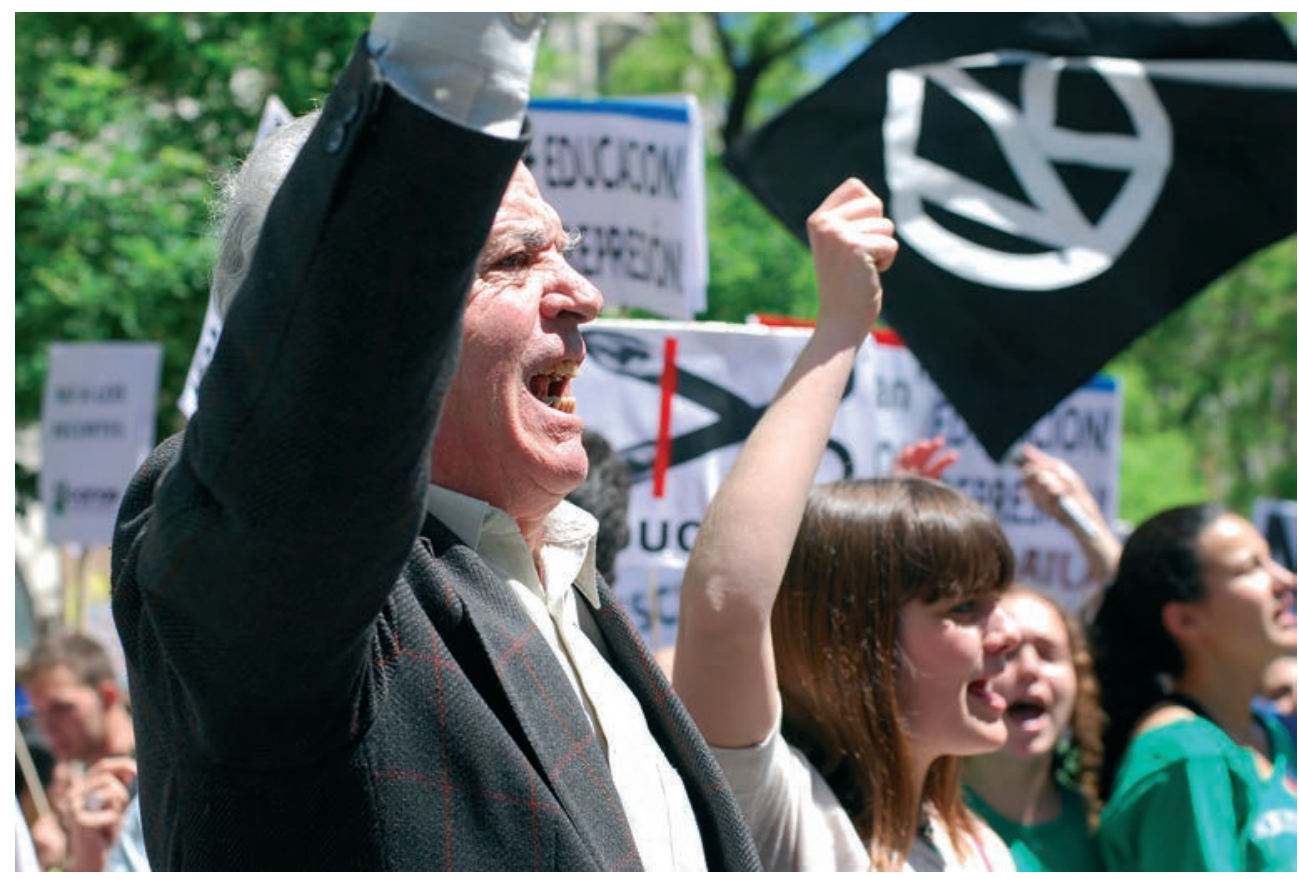

verte con un conocido/amigo/contacto para el primer encuentro con el nuevo contexto. Sonríes mientras ignoras lo que te pasará momentos/días después.

El primer paso, el segundo y todo lo que no se ve, pero hacemos para establecernos en un nuevo lugar, es la experiencia real. Aun así, esta no consta en ninguna parte, solo se intuye, ya que mientras hayas aprendido inglés y trabajado en tu sector, "iya está bien!", dicen.

De hecho, lo mejor que sacas de ello no consta en el currículum. Que sí, mejora, pero ¿qué dice exactamente de ti el hecho de haber estado fuera? Aunque sea obvio, obtienes de cada uno algo diferente y este es el tema: lo que te ha pasado, cómo lo has afrontado (incluido el no hacerlo) y lo que aprendiste de ello es lo que realmente te aporta la experiencia de irte.

Lo primero que hay que tener en cuenta son las circunstancias que te llevan a tomar la decisión de marcharte. Quizás eres, bajo éstas, el único culpable de tomar la decisión e irte. Y rectifico, no es culpa sino sed. Sed de perseguir algo. Sed de buscar el lugar donde puedas hacer aquello que quieres, pese a quien pese, cueste lo que cueste, porque lo tienes claro, sea lo que sea que quieras hacer. En este punto, quisiera insistir que en todo momento me refiero a los jóvenes con estudios o sin ellos que estamos en edad de crecer profesionalmente y labrarnos el futuro, con otras palabras, de pasar a la etapa adulta y asumir responsabilidades.

Pero volviendo a las circunstancias y contexto actual de crisis económica y sin ánimo de ofender, quiero ver la cara positiva y agradecer (sin agradecer a nadie) esta situación que vivimos porque nos ha hecho sentir sed y buscar, contrariamente al camino que habíamos trazado hasta ahora. Me explico: por lo general, nuestra generación ha 
crecido sin faltarle de nada de lo que necesitaba y quería, e incluso en muchos casos sin la consciencia del sacrificio o coste que significaba para quienes nos lo han dado todo.

Y aquí estamos, después de tenerlo todo, hasta un futuro determinado por todo aquello que tenía que venir y por lo que tanto te has esforzado, nada. Tienes nada. $\mathrm{Y}$ es en este punto donde comienza la búsqueda real de aquello que anhelas, tanto si se trata de seguir un camino como el camino en sí mismo.

Así que casi de repente tomas la decisión: te vas. El espacio temporal entre este día y el momento en que partes hacia lo desconcertante es un tiempo en que te vas envalentonando y percatándote de cuántas «últimas veces" harás algo hasta ahora rutinario, al menos por un tiempo. Bueno, quizás no te das cuenta, pero será todo aquello que echarás en falta más temprano que tarde.

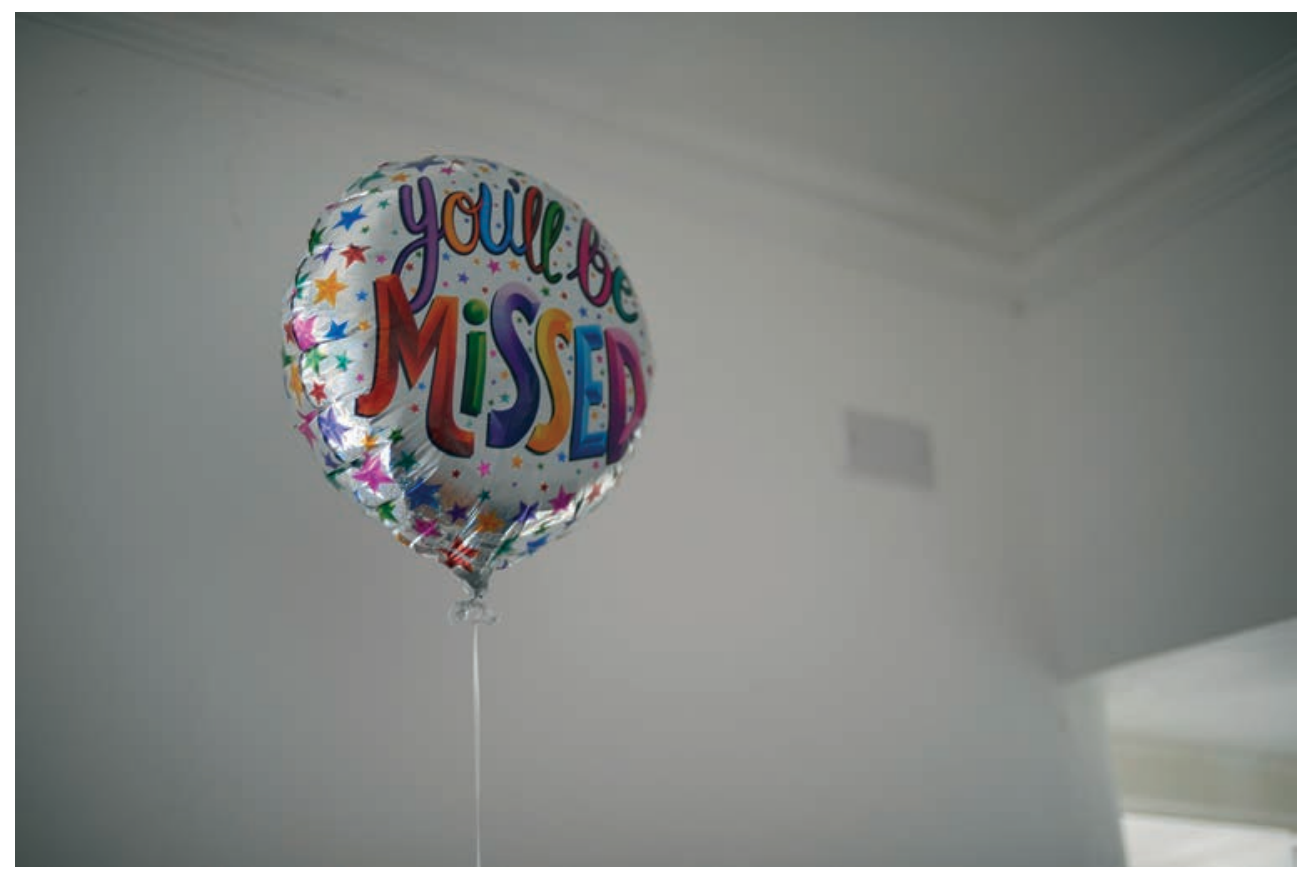

Cuando llegas, no antes, sabes que ya no sueñas ni imaginas. Ahora sí, tu vida real está en tus manos y de nadie más, lo cual fue siempre así, pero tomas conciencia real de ello, muchos por primera vez. Te pasa lo que no imaginas, lo que dentro de la burbuja estable de toda tu vida te hubiera hundido o sido un obstáculo evitable, ahora lo pasas y te hace más fuerte. Te pasa de todo, insisto, bueno y malo, que, si quieres, siempre acaba siendo positivo.

Empiezas a darte cuenta de las prioridades, de lo rápido que van cambiando las de ayer en tu país con las de hoy en el nuevo lugar, las cuales, a su vez, no tienen nada que ver con las de mañana. Un ejemplo claro y básico sería el encontrar sitio donde vivir: empiezas con unos estándares en tu cabeza y acabas aceptando cualquier rincón 
que te parece oro después de lo que has visto, entre lo que te puedes permitir. Un rincón que es tuyo, que representa un gasto fijo, porque no hay que olvidar que, entre emociones y sentimientos, la parte práctica es fundamental para la supervivencia.

Van pasando los días y nada es estable a medio ni largo plazo, pero te acabas sintiendo hasta bien con ello. Seguramente porque estás lejos de casa, lejos de la presión del "cómo tendría que ser todo a tu edad después de todo lo que has estudiado".

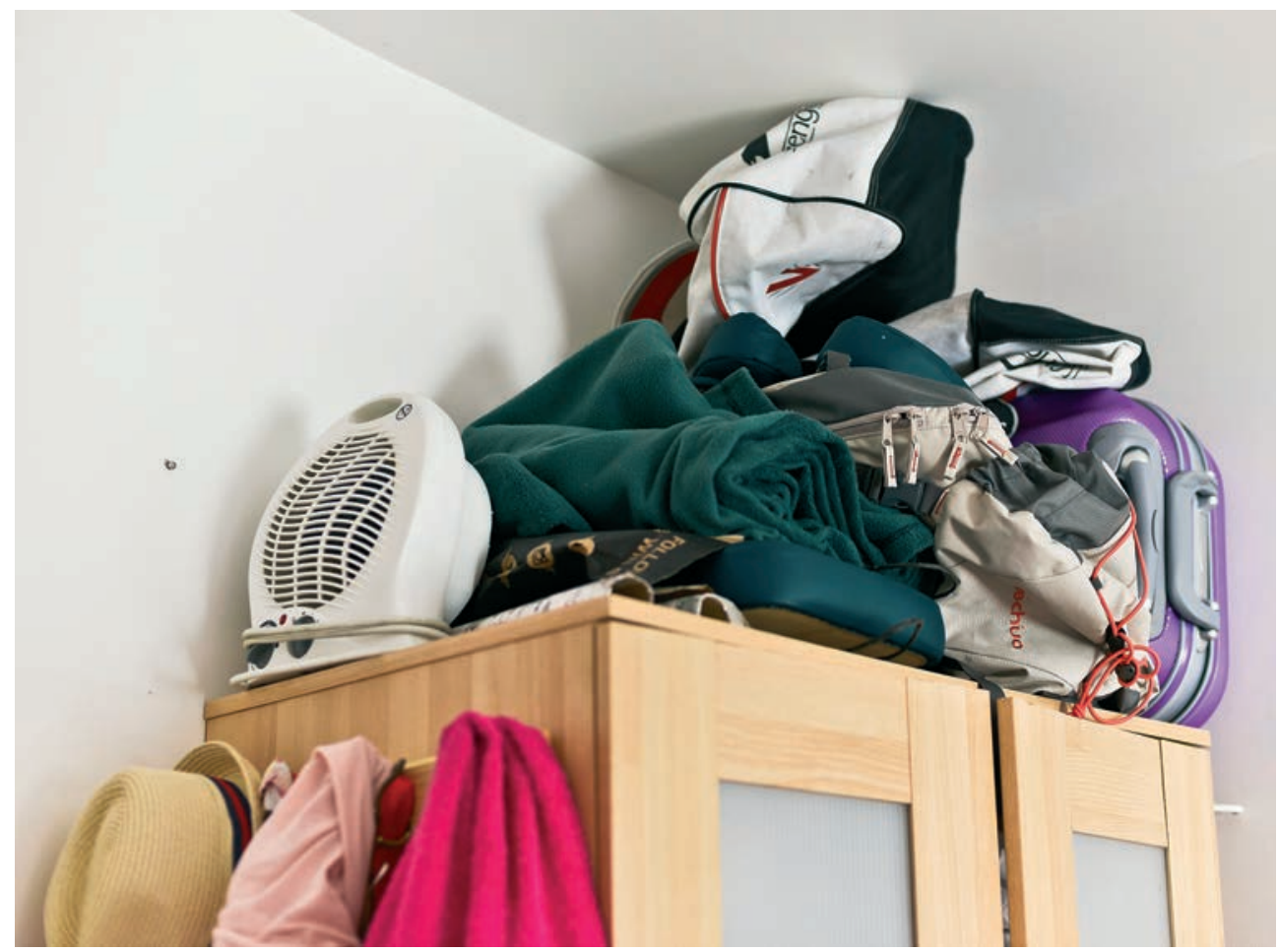

De repente, en el fondo, se abren un sinfín de oportunidades. Oportunidades para aprender, sobre todo de ti mismo. Te toca crecer estrepitosamente. Creces y sientes que la vida solo es ahora, aquí y contigo mismo, que el resto solo te rodea y acompaña, pero no puede sostenerte ahora y, menos, para siempre.

Aceptas un rincón para vivir que decoras con tanta magia como puedes, aceptas trabajos para ir tirando mientras no encuentras algo mejor... y en mi caso, resulta que el "algo mejor" estaba escondido detrás de 6 horas a la semana como nanny, pues la persona que me contrató para cuidar de su hija, al cabo de unos meses necesitaba alguien con conocimientos de delineación de planos para su empresa. De un día para otro empecé como freelance en GB.

Con esto quisiera ejemplificar el hecho de que no hay camino ni resultado exacto para definir el "lograrlo" a la par que todo es tan inimaginable como posible. Poco a poco llega la sensación de ir pisando cada vez más fuerte en ese lugar extraño y donde 
empiezas a sentirte bien, un lugar que nunca es como en casa, pero que empieza a ser tu casa.

Mientras tanto, las prioridades, aunque más lentamente porque todo se va afianzando, siguen cambiando. Cambiando o evolucionando hasta el punto de plantearte el volver. A todos nos ha llegado, antes o después, pero llega. Llega porque en algún momento hay que decidir si a ti, con tus circunstancias y anhelos, te vale la pena. La respuesta no es nada fácil, hay mucho que sopesar en la balanza.

Evidentemente, los hay que tienen clarísimo que su nuevo destino se convirtió en el lugar donde quieren estar, pero a otros, nos pueden las ganas de estar con nuestra gente, el sol, los olores, y de alguna manera, nada compensa aquello que ahora tanto echamos de menos.

Aun así, en tu balanza también pones tus perspectivas laborales. Entonces miras la situación económica en tu país y las oportunidades parecen nulas. Lo parece, pero allí están. No necesitas muchas opciones, necesitas tu oportunidad. La tuya. Esta es la que tienes que perseguir y que, si insistes, conseguirás, porque la buscarás y/o crearás y, si hace falta, aceptarás otra vez un rincón en vez de lujos. Entiéndase el paralelismo: si empezaste desde cero en un lugar nuevo, hasta encontrar un trabajo profesionalmente reconocido y de tu sector, ¿no podría pasar lo mismo en tu tierra donde por encima de todo quieres estar?

Al final, no depende de nada más que de lo que tú quieres y tus prioridades. Personalmente, y por las personas que fui encontrando y conociendo allí, creo que todo depende del motivo que te hizo marchar y de tus objetivos personales, incluyendo en ellos los profesionales.

Cuando te das cuenta de que tú quieres tu futuro en tu tierra, cuando quieres que tu vida transcurra en tu país de origen y, sobre todo, cuando sientes que sea lo que sea que tenga que pasarte quieres que te pase al lado de los tuyos, entiendes que posponer la decisión implica, seguramente, tener más razones para quedarte en el que fue el destino de tu aventura inicial.

Empecé "pisando suelo" como decía una housemate de Londres, y acabé con propuestas grandes y perspectivas muy fascinantes a nivel laboral. Me querían allí, pero la pregunta era: ¿lo que querían para mí es lo que yo quería?

Lo único que tenía claro era mi temor a, en un futuro, estar simbólicamente atada a ese otro país, con un trabajo, quizás una familia, con mi día a día ya establecido al $100 \%$ allí.

Y decidí volver, en el fondo, para evitar atarme más a Londres. Porque la ciudad engancha, la ciudad no para, no duerme y siempre te sorprende. Londres no te lo acabas y las opciones son infinitas. Esto era para todos igual, pero cada uno lo vive y aprovecha de forma distinta según sus necesidades.

Apostar por Londres era sinónimo de encontrar. Los hay quienes encuentran el trabajo de su vida. Los hay que encuentran su pareja y forman una familia. Otros de repente se sienten liberados por ser una ciudad muy pluricultural. Los hay que se quieren quedar, los que volverían, pero no lo hacen y los que lo que encuentran es la certeza de querer volver a su país, a pesar de que las circunstancias que te llevaron a partir no hayan cambiado. Porque el que ha cambiado eres tú y la manera de afrontarlo. 
Finalmente regresé, por probar, con la idea tranquilizadora de que siempre podía volver a marchar, pues ya sabía lo que implicaba y, por encima de todo, me conocía capaz. No hay que olvidar que volver es otro salto al vacío y de duración indefinida, es decir, un tiempo de incertidumbre en el que dudas de la decisión que tomaste ya que, a pesar de todo lo que echabas de menos, tenías en tus manos el inicio de una carrera profesional (aquello tan deseado).

Pero diste el salto. Regresaste a tu tierra con un nuevo aprendizaje, un tú mejorado que de repente tiene que volver a encajar en el lugar de donde vienes y donde todo estaba aprendido. Eso que recordabas y tenías ganas de revivir de nuevo, también ha cambiado. También hay cosas que se quedan intactas y te sientes afortunado. Comienzas desde cero, otra vez, pero en un entorno conocido e incluso previsible, lo cual se convierte en una hazaña tanto o más difícil que hacerlo en uno del que desconoces cómo es todo.

Sobrevivir otra vez entre emociones y sentimientos. Búscate la vida. Dudas y te reafirmas constantemente. Sigues, insistes y pones como condición laboral prioritaria el lugar donde quieres estar, por delante incluso del sueldo, los horarios, etc. Socialmente cuesta que se entienda, por lo general. Pero tú lo tienes claro, lo has vivido y nada te merece más que estar aquí.

Llega también el momento de dejar las dudas atrás y trabajar con determinación por lo que quieres, para que la oportunidad te encuentre trabajando. Es fácil tildar de utópico este mensaje, pero entonces me pregunto: ¿por qué fuera todo es posible y aquí no? No hablo cuantitativamente de los buenos o malos sueldos o trabajos. No hablo de lo que es palpable sino del hecho de que desistamos y lo permitamos entre todos.

Llegados a este punto, quisiera apuntar que, a pesar de que se deposite toda la fe en uno mismo y en sus ganas para que las cosas pasen, esto no exime a las políticas de estado de invertir en mejorar para adaptarse a los tiempos y a crecer como país.

Pero volviendo a la, por muchos, menospreciada utopía, de que el sistema sea más o menos adecuado a ti, seguirá siendo solo una circunstancia más en tu vida que condiciona, pero no determina.

Por lo tanto, supongo que lo más importante es tener claras las prioridades, aceptando que cambian para decidir en cada momento y, sobre todo, las ganas y paciencia para vivir todo aquello que la vida te traiga mientras caminas persiguiendo tus objetivos y sueños, a corto o largo plazo. Y esto no entiende de marcharse fuera o no. Ni todos tenemos que hacerlo ni hacerlo es la clave del éxito.

Quien lo hizo o lo hará, tanto partir como volver, es porque siente que debe, motivado por un largo etcétera de razones que te llevan a sobrevivir de las más dulces a las más amargas aventuras.

La aventura de volver duró 6 meses que es el tiempo que tardé en encontrar el trabajo que quería, sabiendo que no sería indefinido pero que me permitiría ir y crecer en mi camino. Resulta que lo que parecía imposible estaba pasando: trabajar como arquitecta técnica, un sector altamente castigado, en la ciudad donde quería vivir y con las tareas y valores de la profesión por los que, creía, tenía habilidad.

Quizás marchamos porque aquí no encontramos lo que queremos. No pasa nada, formará parte de nuestro camino. 
Después de todo, la oportunidad más grande que te pueden dar los que te lo quieren dar todo es la de conocerte a ti mismo y no tener miedo de hacerlo, para saber realmente qué quieres y luchar por ello. Para algunos esto implica irse, para otros no, e irse te da lo que necesitas, aunque no lo busques, como tantas otras cosas que te sucedieron y te sucederán en la vida.

Fecha de recepción: 20 de diciembre de 2016*

Fecha de aprobación: 12 de abril de 2017

\footnotetext{
* Las fechas de recepción y aprobación vienen referidas al conjunto de la sección TEMAS EMERGENTES.
} 